\title{
Development of Pseudo-random Binary Arrays for Calibration of Surface Profile Metrology Tools
}

\author{
Samuel K. Barber, ${ }^{\text {a) }}$ Paul Soldate, ${ }^{\text {b) }}$ Erik H. Anderson, ${ }^{\text {a) }}$ Rossana Cambie, ${ }^{\text {a) }}$ \\ Wayne R. McKinney, ${ }^{\text {a) }}$ Peter Z. Takacs, ${ }^{\text {c) }}$ Dmytro L. Voronov, ${ }^{\text {a) }}$ Valeriy V. \\ Yashchuk $^{\text {a) }}$ \\ a) Lawrence Berkeley National Laboratory, Berkeley, California, 94720 \\ b) Rensselaer Polytechnic Institute, Troy, New York, 12180 \\ c) Brookhaven National Laboratory, Upton, New York 11973
}

\begin{abstract}
Optical Metrology tools, especially for short wavelength (EUV and X-Ray), must cover a wide range of spatial frequencies from the very low, which affects figure, to the important mid-spatial frequencies and the high spatial frequency range, which produces undesirable scattering. A major difficulty in using surface profilometers arises due to the unknown Point-Spread Function (PSF) of the instruments [1] that is responsible for distortion of the measured surface profile. Generally, the distortion due to the PSF is difficult to account because the PSF is a complex function that comes to the measurement via the convolution operation, while the measured profile is described with a real function. Accounting for instrumental PSF becomes significantly simpler if the result of measurement of a profile is presented in a spatial frequency domain as a Power Spectral Density (PSD) distribution [2]. For example, the measured PSD distributions provide a closed set of data necessary for three-dimensional calculations of scattering of light by the optical surfaces [3], [4]. The distortion of the surface PSD distribution due to the PSF can be modeled with the Modulation Transfer Function (MTF), which is defined over the spatial frequency bandwidth of the instrument [1], [2]. The measured PSD distribution can be presented as a product of the squared MTF and the ideal PSD distribution inherent for the System Under Test (SUT). Therefore, the instrumental MTF can be evaluated by comparing a measured PSD distribution of a known test surface with the corresponding
\end{abstract}


ideal numerically simulated PSD. The square root of the ratio of the measured and simulated PSD distributions gives the MTF of the instrument.

In previous work [5], [6] the instrumental MTF of a surface profiler was precisely measured using reference test surfaces based on Binary Pseudo-Random (BPR) gratings. Here, we present results of fabricating and using two-dimensional (2D) BPR arrays that allow for a direct 2D calibration of the instrumental MTF. BPR sequences are widely used in engineering and communication applications such as Global Position System, and wireless communication protocols. The ideal BPR pattern has a flat "white noise" response over the entire range of spatial frequencies of interest. The BPR array used here is based on the Uniformly Redundant Array prescription [7] initially used for X-ray and gamma ray astronomy applications. The URA's superior imaging capability originates from the fact that its cyclical autocorrelation function very closely approximates a delta function, which produces a flat PSD.

Three different size BPR array patterns were fabricated by electron beam lithography and ICP etching of silicon. The basic size unit was $200 \mathrm{~nm}, 400 \mathrm{~nm}$, and 600 $\mathrm{nm}$. Two different etch processes were used, $\mathrm{CF}_{4} / \mathrm{Ar}$ and $\mathrm{HBr}$, which resulted in undercut and vertical sidewall profiles, respectively.

The 2D BPR arrays were used as standard test surfaces for MTF calibration of the MicroMap ${ }^{\mathrm{TM}}-570$ interferometric microscope using all available objectives. The $\mathrm{HBr}$ etched two-dimensional BPR arrays have proven to be a very effective calibration standard making possible direct calibration corrections without the need of additional calculation considerations, while departures from the ideal vertical sidewall require an additional correction term for the $\mathrm{CF}_{4} / \mathrm{Ar}$ etched samples. [8] Initial surface roughness of low cost "prime" wafers limits low magnification calibration but should not be a limitation if better polished samples are used.

\section{Introduction}


A key challenge in the developing high performance optical systems, especially at short wavelengths for EUV and X-Ray applications, is the accurate measurement of the optical surface profile over a very wide range of spatial frequencies from the macroscopic size of the optic to the nanometer regime. Measurements from different tools, optimized for low, mid, and high spatial frequencies must be combined to give the necessary information for quantitative understand and modeling. Therefore accurate calibration of individual tools and cross calibrations between tools is very important. Surface profilometers, such as interferometric microscopes have become the basic tool for determining the mid spatial frequencies ( $\mathrm{mm}$ to $\mu \mathrm{m}$ ) while atomic force microscopes provide the high-spatial frequency information. Interferometric microscopes routinely are used to characterize high-quality optical surfaces that have sub-Angstrom (rms) roughness. Unfortunately, these instruments have non-unity Modulation Transfer Functions (MTF) that roll-off at the higher spatial frequencies. Some of the sources for this roll-off are the optical system itself (lens NA etc), detector response (pixel size, shape, amplifier bandwidth) signal processing algorithms and environmental factors (i.e. drift and vibrations). While theoretically the individual contributions to the MTF might be characterized and combined to estimate the MTF, these contributions are generally difficult to account for separately. In practice, the entire instrument MTF is determined by measuring a reference surface with a well-known surface profile. A "white noise" measurement technique can be used with a reference sample, which includes a wide range of spatial frequencies in one pattern. The square root of the ratio of the power spectral density (PSD) of the instrument to the PSD of the reference surface profile is the instrument MTF. Using modern nanofabrication methods, near arbitrary two-dimensional patterns can be fabricated at the length scale of interest to calibrate interferometric microscopes. The pattern should be moderately easy to calculate and have a uniform PSD throughout the spectral range of interest. To meet these requirements, a test surface consisting of a two-dimensional binary pseudo-random array (BPRA) based on uniformly redundant arrays (URAs) has been selected and represents the two dimensional extension to the previous grating methods. URA patterns have been extensively studied for a wide range of applications and have the mathematical property of an extremely sharp periodic auto-correlation function with very low and uniform side lobes. Due to the Fourier 
transform relationship between the PSD and the auto-correlation function (WienerKinchin theorem), the URA pattern has a flat PSD. While modern mathematical theory is used in the proving of URA properties [9], the actual prescription for calculating the URA is computationally straightforward.

\section{BPRA properties}

Since the approach to pattern the BPRA is to use electron beam lithography, where almost arbitrary patterns can be generated, the difficulty is in choosing the "best" pattern. The pattern should be moderately easy to calculate, produce reasonable size datasets, and have a flat power spectral density up to the spatial frequency of interest to provide uniform calibration accuracy over the entire spatial frequency range. Sequences with sharp autocorrelation functions will have wide bandwidth flat PSDs by nature of the Wiener-Khinchin theorem. Early work in communications theory produced a set of sequences [10] named for the author R. H. Barker, which have the property that the nonorigin autocorrelation terms are either 0 or 1 . These sequences are widely used in engineering applications including wireless communication and even e-beam correlation alignment algorithms. [11] However, sequences of only up to 13 elements are known with this property. The BPRA needs several thousand elements so a different sequence is needed. The two-dimensional URA prescription as described by E. E. Fenimore and T. M. Cannon [7] has the property that the cyclical autocorrelation function is a delta function with a small constant offset. The prescription is easily calculated by the formula in the reference. Figure 2 shows an example of a 43 by 41 URA pattern and the corresponding cyclical autocorrelation function. Figure 4 shows an SEM micrograph of part of the URA pattern used as a metrology reference surface.

\section{Fabrication of BPRA}

Ideally the top and bottom surfaces of a BPRA should have identical reflectivity. Using etching of a single solid substrate is a straightforward way to satisfy this requirement. Further, the sample should be stable, easy to handle, withstand storage and shipping from instrument to instrument. Our approach is to etch the pattern into a single crystal silicon substrate. Provided that the etch process is sufficiently uniform across the area of interest and is not correlated with the feature size, the BPRA will have the desired 
surface profile. Electron beam lithography with modest resolution resists easily meets the linewidth requirements of $200 \mathrm{~nm}, 400 \mathrm{~nm}$ and $600 \mathrm{~nm}$ basic structures. The process can be extended to both larger and smaller structures with the use of optimized resolution/throughput e-beam resists. The process flow is as follows:

1. Prepare a 4-inch wafer with HMDS adhesion promoter.

2. Spin a chemically amplified positive resist, TOK cap 138 and softbake at 130 C for 90 seconds.

3. Expose the BPRA pattern using $100 \mathrm{KeV}$ electron beam lithography, about $30 \mu \mathrm{C} / \mathrm{cm}^{2}$ dose.

4. Bake at $110 \mathrm{C}$ for 90 seconds for the post exposure bake.

5. Develop in TMAH base developer (LDD26W) for 60 seconds.

After development, the sample is ready for etch pattern transfer in an Induction Coupled Plasma (ICP) etcher (Oxford Instruments Plasma lab 150). Two etch chemistries were used. The first, based on $\mathrm{SF}_{6}$ and Argon, is reliable and fast but does not produce vertical sidewalls. The parameters for the etch are as follows: $4 \mathrm{sccm} \mathrm{SF} 6$ $7 \mathrm{sccm}$ Ar, $6 \mathrm{mTorr}$ chamber pressure, RF power $30 \mathrm{~W}$, ICP power $1500 \mathrm{~W}$, temperature 0C. Figure 1 shows the SEM and AFM of the etched BPRA array with this chemistry. The undercut was sufficient that it could not be ignored but not enough to prevent the BPRA from being used as a reference test surface for instrument calibration. A small correction term was added to the MTF to account for the undercut. A second etch chemistry was used to generate better vertical sidewall profiles. The parameters for that etch are as follows: $20 \mathrm{sccm} \mathrm{HBr}, 5 \mathrm{~m}$ Torr chamber pressure, RF power $60 \mathrm{~W}$, ICP power $700 \mathrm{~W}$, temperature $25 \mathrm{C}$. Figure 2 shows an SEM and AFM micrograph of the etched BPRA. The sidewall is essentially vertical producing near ideal structures for a reference sample. No evidence of feature size etch dependent etch rates were observed at the dimensions of interest. The optimum etch depth is approximately $120 \mathrm{~nm}$, though not critical. The etch depth over the small $\mathrm{mm}$ size pattern in the center of the wafer was essentially uniform.

\section{Experimental results}


BPRA patterns with three fundamental sizes $(200 \mathrm{~nm}, 400 \mathrm{~nm}$, and $600 \mathrm{~nm})$ were exposed and etched with both $\mathrm{HBr}$ and $\mathrm{SF}_{6}$.

Figure 5 shows a typical result of the BPRA surface height measurement with the Micromap ${ }^{\mathrm{TM}}-570$ interference microscope. Even by eye, the smallest features (higher spatial frequency components) have a reduced amplitude but the AFM and SEM data shows that the etch depth is constant across feature size. The roll-off of the instrumental MTF therefore needs correction in order to accurately represent the actual surface profile. The comparison of the undercut $\mathrm{SF}_{6}$ etched samples with the vertical sidewall $\mathrm{HBr}$ etched samples is shown in figure 6. For ease of visualization, the 2D PSD's are integrated along the $\mathrm{X}$ and $\mathrm{Y}$ directions to produce two 1D PSD's along the $\mathrm{Y}$ and $\mathrm{X}$ directions, respectively. Both samples have fairly similar profiles with a flat low spatial frequency region and a steep roll off in the high frequency range that is a signature of the instrumental MTF. The difference due to the sloped sidewall is small, approximately $10 \%$ and not easily observed with the data plotted in a logarithmic format.

Note, however, the existence of the raised low spatial frequency tails in the PSD's from the $2.5 \times$ and $5 \times$ objectives. These distortions are due to surface variations in the silicon wafer. Even etch depths of $\sim 100 \mathrm{~nm}$ are not large enough to suppress the influence of low frequency variations of the wafer surface. To further illustrate this point an un-processed area of the wafer was measured with the $2.5 \times$ objective and compared with a $600 \mathrm{~nm}$ BPRA that was etched to only $49 \mathrm{~nm}$. Figure 7 shows the comparison. The peek to valley roughness of the low cost "prime" silicon wafer is about $10 \mathrm{~nm}$, which is a significant fraction of the $49 \mathrm{~nm}$ etch depth. As a consequence the low spatial frequencies have additional errors originating from the wafer itself. A solution to this problem is to use a higher quality polished silicon substrates, which are commercially available.

Because of the nearly ideal shape of the array elements with the $\mathrm{HBr}$ etched samples, the procedure for determining the MTF requires no additional modeling to account for the finite sidewall slope and is drastically simplified compared to the procedure for the $\mathrm{SF}_{6}$ samples. The PSD inherent to each of the samples is assumed to be flat out to the Nyquist frequency of the BPRA. Thus, to determine the MTF we simply need to divide the measured 2D PSD by a flat 2D PSD with an appropriate constant 
value. The square root of the ratio gives the MTF of the instrument. The value of the "white noise" PSD is experimentally determined by the low frequency components of the measured PSD. For these BPRA's, we expect the value of the PSD to be the same over the entire frequency range using the value of the measured 2D PSD in the low frequency range to construct the theoretical flat 2D PSD inherent for the BPRA. Using a single value of the PSD at the lowest frequency is problematic since any single data point could be influenced by random noise. A better approach is to average the PSD over a range of frequencies to reduce random noise effects. However, as frequency range increases, the MTF starts to distort the PSD, which will adversely affect the determination of the theoretical PSD amplitude. So, in order to determine an accurate value for the amplitude of the theoretical flat PSD, the frequency range over which to average the measured PSD should be large enough to average out random noise effects, but not too large as to incorporate the MTF distortions at the higher frequencies. Since the determination of this frequency range is somewhat arbitrary, it is important to examine the effects of using different frequency ranges in determining the theoretical 2D PSD amplitude.

Using the above procedure, the MTF of the Micromap ${ }^{\mathrm{TM}}-570$ has been measured for the following objectives: $5 \times, 10 \times$ and $20 \times$. For the first two corrections $(5 \times$ and $10 \times)$, the MTF is determined from measurements of the $600 \mathrm{~nm}$ BPRA's. For the latter $(20 \times)$ the calibration used the $400 \mathrm{~nm}$ BPRA's. These choices were made based on the effective detector pixel sizes for the given objectives. It is advantageous to have the effective detector pixel size close to the elementary pitch of the BPRA, but not any smaller. When the effective detector pixel size is smaller, then the detector samples at a frequencies higher than the Nyquist frequency of the BPRA. If the effective pixel size is much larger than the elementary pitch, the overall number of the elements of a BPRA with the size that is large enough to cover the instrumental field of view appears to be very huge. Fabrication of such a BPRA would be significantly more challenging. Thus, for the $5 \times$ and $10 \times$ objectives, which correspond to detector pixel sizes of $1.92 \mu \mathrm{m}$ and $0.96 \mu \mathrm{m}$, respectively, we chose the $600 \mathrm{~nm}$ BPRA's. And for the $20 \times$ objective $(0.49 \mu \mathrm{m})$ we use the 400 nm BPRA's.

Once the MTF has been determined for a given objective, it can be used to correct the measurements made with the same objective. The effectiveness of the MTF 
calibration from the PSD's obtained after correcting the BPRA measurements is gauged by observing whether the corrected PSD of another BPRA is flat across the entire spatial frequency range giving confidence that the correction allows accurate extraction of the PSD inherent from the surface.

The above procedure was applied to both the $10 \times$ and $20 \times$ objectives. Figure 8 shows the $a b$ into MTF calibration of the $20 \times$ objective $(0.49 \mu \mathrm{m}$ effective pixel size $)$ of two BPRA patterns using a third as the reference. The lower curve is the un-corrected data and the top curve has the correction factor applied. The corrected response is largely flat, an indication of success. Only in Figs. $8 \mathrm{~b}$ and $8 \mathrm{c}$ are there noticeable deviations from a flat PSD at higher frequencies. In these cases there appears to be a slight upward tail at higher spatial frequencies. Most likely imperfections like those seen in Figs. 8b and 8c arise due to aliasing effect [1]. Additionally, the environmental conditions during the measurements that were used to determine the MTF could be different from those made during subsequent measurements made hours or days later. This suggests that in order to make the best use of these calibration surfaces, they should be measured before and after an actual measurement to obtain two versions of the MTF. The two MTF's should then be averaged before correcting the actual measurement in order to average out the effect of the varying environmental conditions.

Figure 9 shows similar results of MTF calibration obtained using the $10 \times$ objective. The results of the $10 \times$ calibration are very similar to those made with the $20 \times$ calibration which provides further validation of the proposed MTF calibration method using pseudo-random arrays.

\section{Conclusion}

BPRA samples suitable for calibration of optical metrology tools have been designed based on the URA prescription and etched into silicon. The straight sidewall etch, using $\mathrm{HBr}$, results in a square wave profile which requires no additional correction terms based on SEM and AFM measurement of the element shape. Using the HBr etched

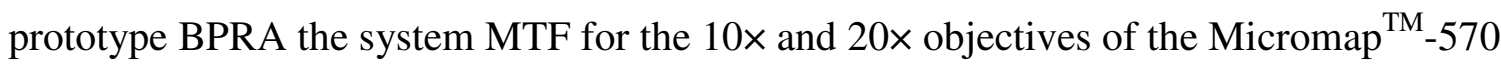
interferometric microscope has been experimentally measured. Because of the known problem of low frequency waviness of "prime" 0.5 -mm-thick wafers, the samples were 
found to be less suitable for an MTF calibration of the $2.5 \times$ or $5 \times$ objectives but a better polished starting sample is expected to address this issue.

The general technique of using a "white noise" test methodology based on special array prescriptions such as the URA can be extended to both higher and lower spatial frequency measurement tools and represents a practical method for accurate cross calibrations.

\section{Acknowledgements}

The authors are grateful to Howard Padmore for useful discussions and Dawn Hilken for nanofabrication. The Advanced Light Source is supported by the Director, Office of Science, Office of Basic Energy Sciences, Material Science Division, of the U.S. Department of Energy under Contract No. DE-AC02-05CH11231 at Lawrence Berkeley National Laboratory. Certain commercial equipment, instruments, or materials are identified in this document. Such identification does not imply recommendation or endorsement by the US Department of Energy, LBNL, or ALS, nor does it imply that the products identified are necessarily the best available for the purpose.

[1] G. D. Boreman, "Modulation Transfer Function in Optical and Electro-optical Systems,” SPIE Press, Bellingham (2001).

[2] J. W. Goodman, Introduction to Fouriew Optics. Third edition (Roberts \& Company, Englewood, Colorado, 2005).

[3] E. L. Church, H. A. Jenkinson, and J. M. Zavada, "Relationship between surface scattering and micro-topographic features," Opt. Eng. 18(2), 125-36 (1979).

[4] J. C. Stover, "Optical Scattering," second edition, SPIE Optical Engineering Press, Bellingham (1995). 
[5] V. V. Yashchuk, W. R. McKinney, P. Z. Takacs, "Binary pseudo-random grating as a standard test surface for measurement of modulation transfer function of interferometric microscopes," Proc. SPIE 6704, 670408 (2007).

[6] Valeriy V. Yashchuk, Wayne R. McKinney, and Peter Z. Takacs, Binary Pseudorandom Grating Standard for Calibration of Surface Profilometers, Optical Engineering 47(7), 073602-1-5 (2008).

[7] E. E. Fenimore and T. M. Cannon, "Coded aperture imaging with uniformly redundant arrays", Applied optics 17(3), 337-47 (1978).

[8] Samuel K. Barber, Paul Soldate, Erik Anderson, Rossana Cambie, Wayne R. McKinney, Peter Z. Takacs, Dmytro L. Voronov, Valeriy V. Yashchuk, Binary Pseudo-random Gratings and Arrays for Calibration of the Modulation Transfer Function of Surface Profilometers: Recent Developments, Abstract to Optics and Photonics 2009: Optical Engineering and Applications Symposium. (San Diego, August 02-06, 2009).

[9] A. Busboom, H. Elders-Boll, and H.D. Schotten, "Uniformly Redundant Arrays" Experimental Astronomy, 8, 97-123, (1998)

[10] R. H. Barker, "Group Synchronizing of Binary Digital Sequences." Communication Theory". London: Butterworth, pp. 273-287, (1953).

[11] E.H. Anderson, "Specialized Electron Beam Nano-Lithography for EUV and X-Ray Diffractive Optics”, IEEE J. Quan. Electr. 42, 1, (Jan. 2006).

\section{List of figures}

Figure 1. SEM(a) and AFM (b) micrographs of the $\mathrm{SF}_{6} / \mathrm{Ar}$ etched BPRA samples. The departure from vertical sidewalls reduces the highest spatial frequencies and requires a small correction in the MTF calculation.

Figure 2. AFM and SEM micrographs of the HBr etched BPRA samples. The departure from vertical sidewalls is small enough to be ignored in the MTF calculation.

Figure 3 . The 43 by 41 URA pattern as calculated by the prescription of E. E. Fenimore and T. M. Cannon (a) and sharp, cyclical autocorrelation with constant sidelobe (b). 
Figure 4. SEM micrograph of a section of the URA pattern used as the reference surface.

Figure 5. Micromap ${ }^{\mathrm{TM}}-570$ interference microscope surface height measurement with the $50 \times$ objective $(0.19 \mu \mathrm{m}$ effective pixel size $)$ of the $200 \mathrm{~nm}, \mathrm{SF}_{6}, \mathrm{BPRA}$ with etch depth of $122 \mathrm{~nm}$. The area shown is $125 \mu \mathrm{m}$ by $94 \mu \mathrm{m}$.

Figure 6. The comparison of the undercut sidewall $\mathrm{SF}_{6}$ etched sample (a) with the essentially vertical sidewall $\mathrm{HBr}$ sample (b). Even with the $\mathrm{SF}_{6}$ etched BPRA, the PSD of the surface deviates from flat by at most $\sim \% 10$. Note that the $50 \times$ objective data is not available for the $\mathrm{HBr}$ etched sample. For the objectives measured, the $\mathrm{HBr}$ etched BPRA has essentially an ideal profile and flat PSD beyond the frequencies of interest.

Figure 7 Micromap ${ }^{\mathrm{TM}}-570$ measurements with the $2.5 \times$ objective of the bare silicon surface (a) and shallow $49 \mathrm{~nm}$ etch depth $600 \mathrm{~nm}$ BPRA (b). The large-scale roughness of a standard silicon "prime" wafer is evident at lower spatial frequencies. The low frequency roughness limits the use of these reference samples in calibrating the $2.5 \times$ and $5 \times$ objectives for high accuracy. Polished silicon flats should not have this roughness.

Figure 8. Ab Initio MTF Calibration 20× objective $(0.49 \mu \mathrm{m}$ effective pixel size) of two BPRA patterns using a third pattern as the reference summed to show the $\mathrm{X}$ (left) and $\mathrm{Y}$ (right) PSD. The lower curve is un-corrected and the top curve has the correction factor applied. The corrected response is largely flat.

Figure 9. Ab Initio MTF Calibration 10× objective (0.96 $\mu \mathrm{m}$ effective pixel size) of two BPRA patterns using a third pattern as the reference summed to show the $\mathrm{X}$ (left) and $\mathrm{Y}$ (right) PSD. The lower curve is un-corrected and the top curve has the correction factor applied. The corrected response is largely flat. 


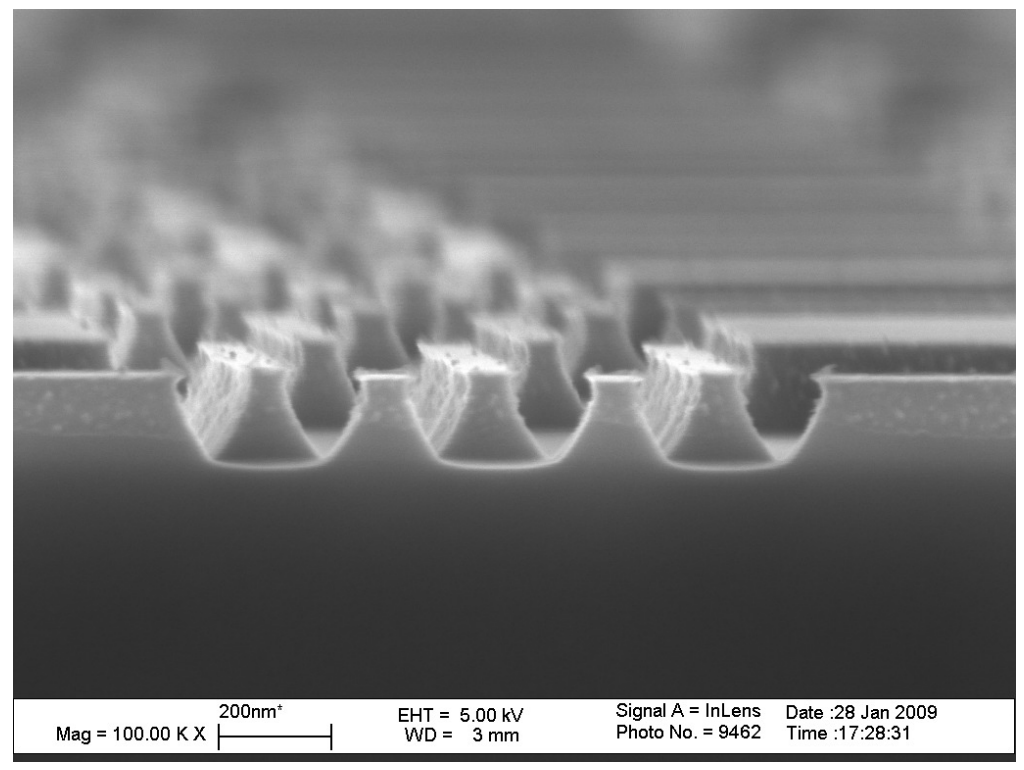

(a)

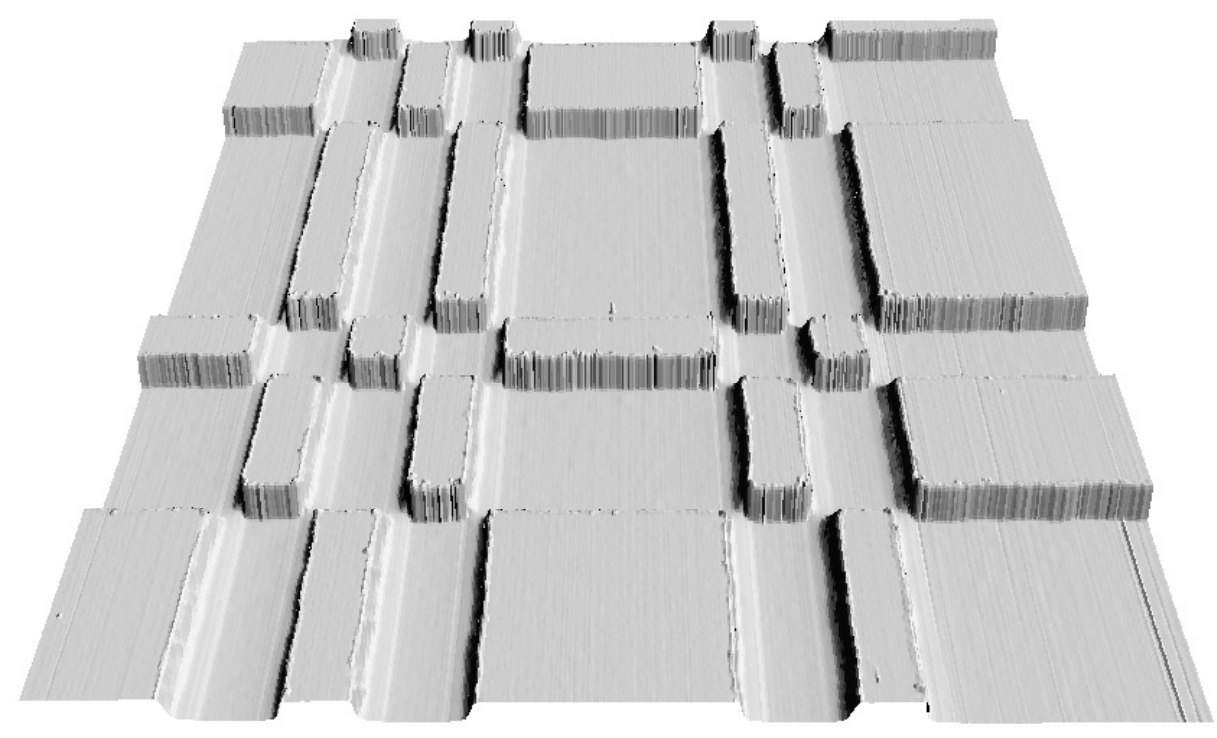

(b)

Figure 1. SEM(a) and AFM (b) micrographs of the $\mathrm{SF}_{6} / \mathrm{Ar}$ etched BPRA samples. The departure from vertical sidewalls reduces the highest spatial frequencies and requires a small correction in the MTF calculation. 

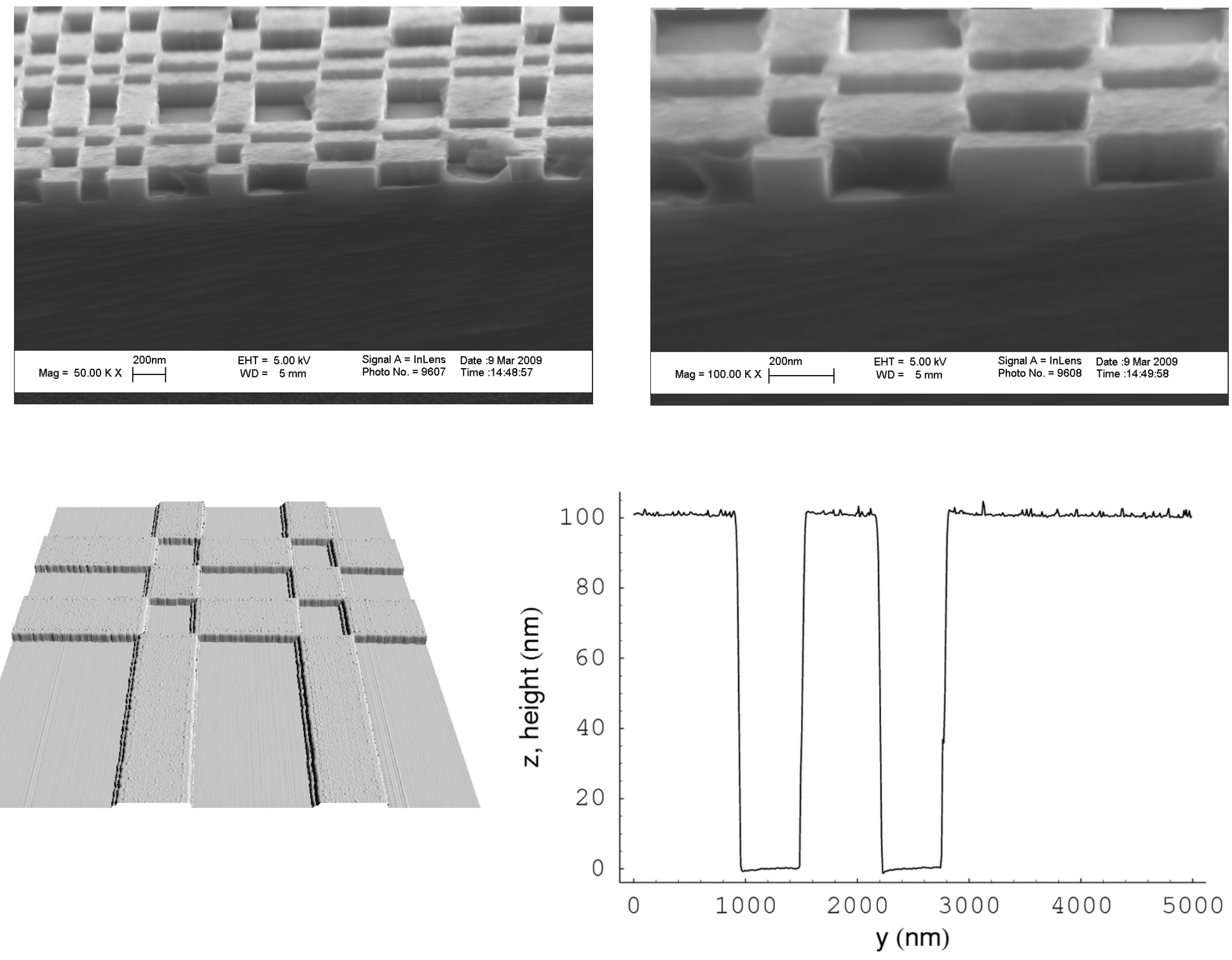

Figure 2. AFM and SEM micrographs of the $\mathrm{HBr}$ etched BPRA samples. The departure from vertical sidewalls is small enough to be ignored in the MTF calculation. 


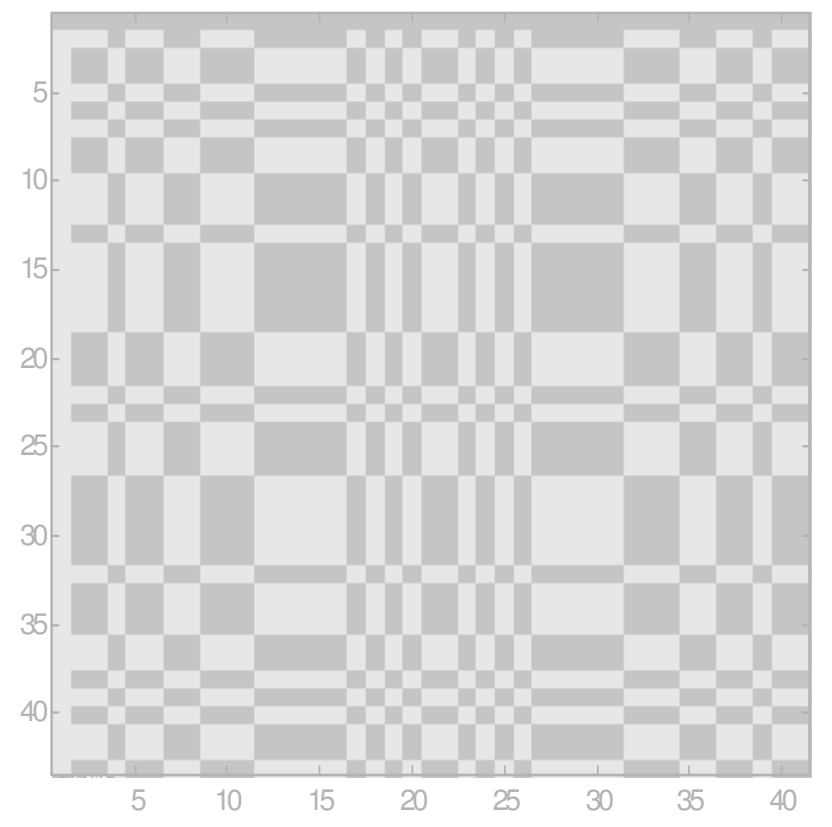

(a)

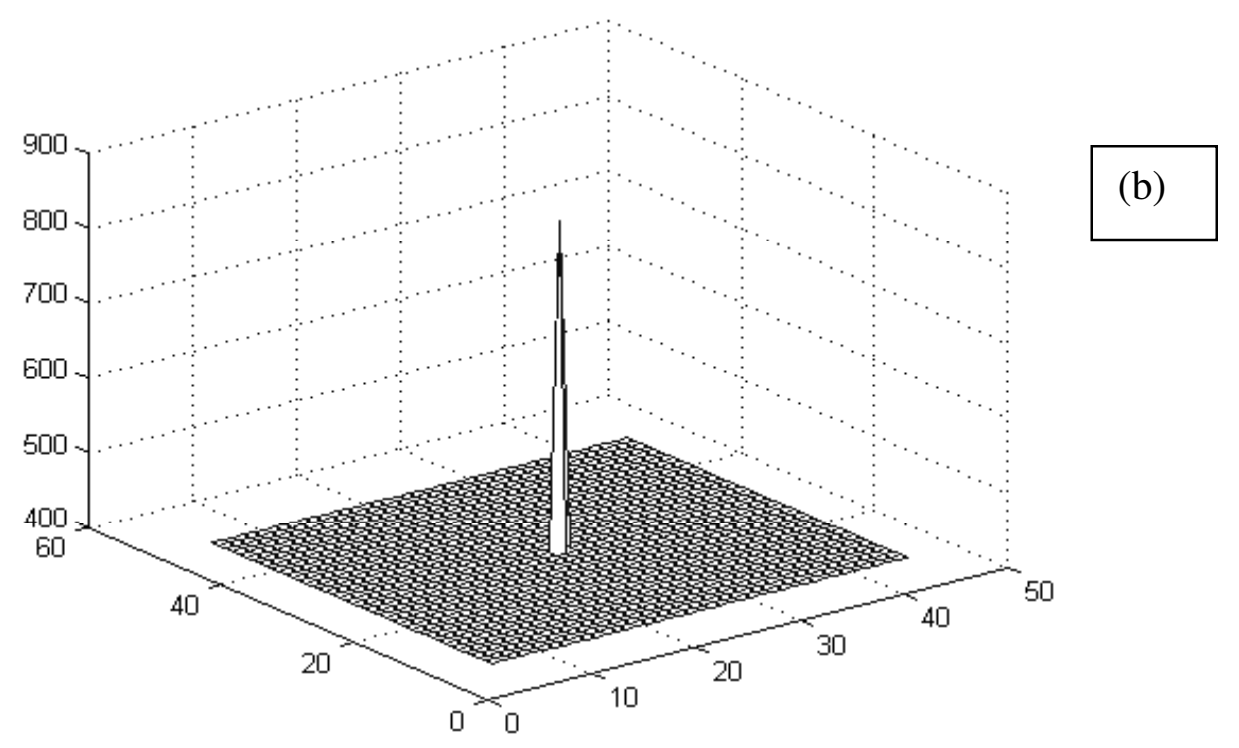

Figure 3. The 43 by 41 URA pattern as calculated by the prescription of E. E. Fenimore and T. M. Cannon (a) and sharp, cyclical autocorrelation with constant sidelobe (b). 


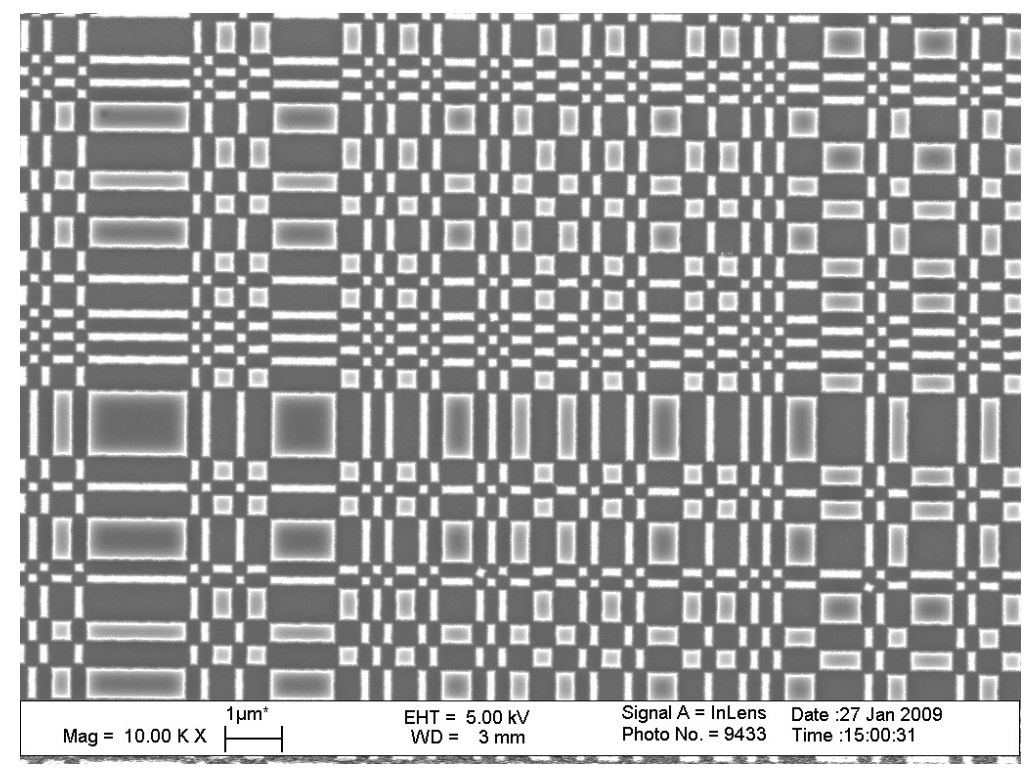

Figure 4. SEM micrograph of a section of the URA pattern used as the reference surface. 


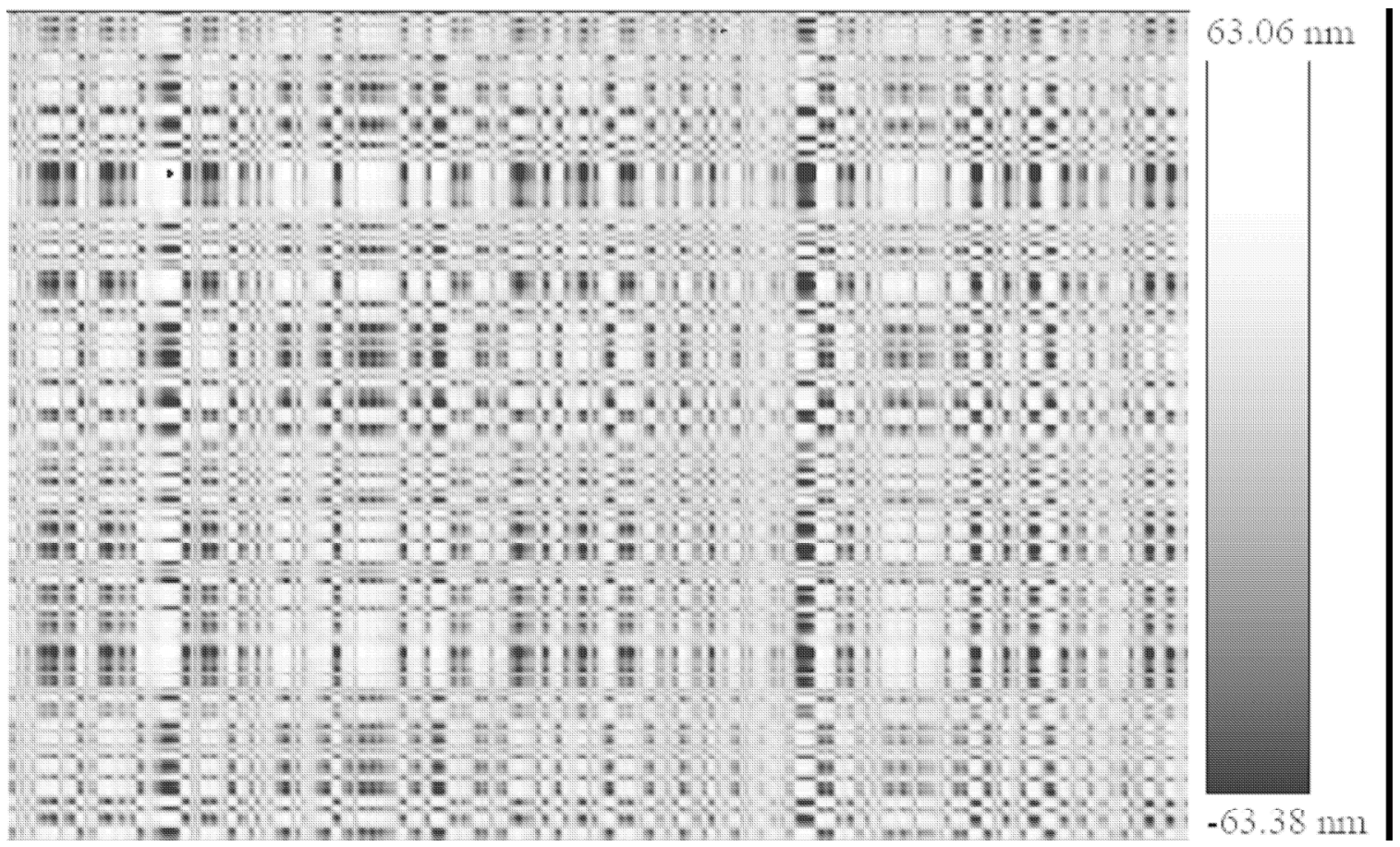

Figure 5. Micromap ${ }^{\mathrm{TM}}-570$ interference microscope surface height measurement with the $50 \times$ objective $(0.19 \mu \mathrm{m}$ effective pixel size $)$ of the $200 \mathrm{~nm}, \mathrm{SF}_{6}$, BPRA with etch depth of $122 \mathrm{~nm}$. The area shown is $125 \mu \mathrm{m}$ by $94 \mu \mathrm{m}$. 

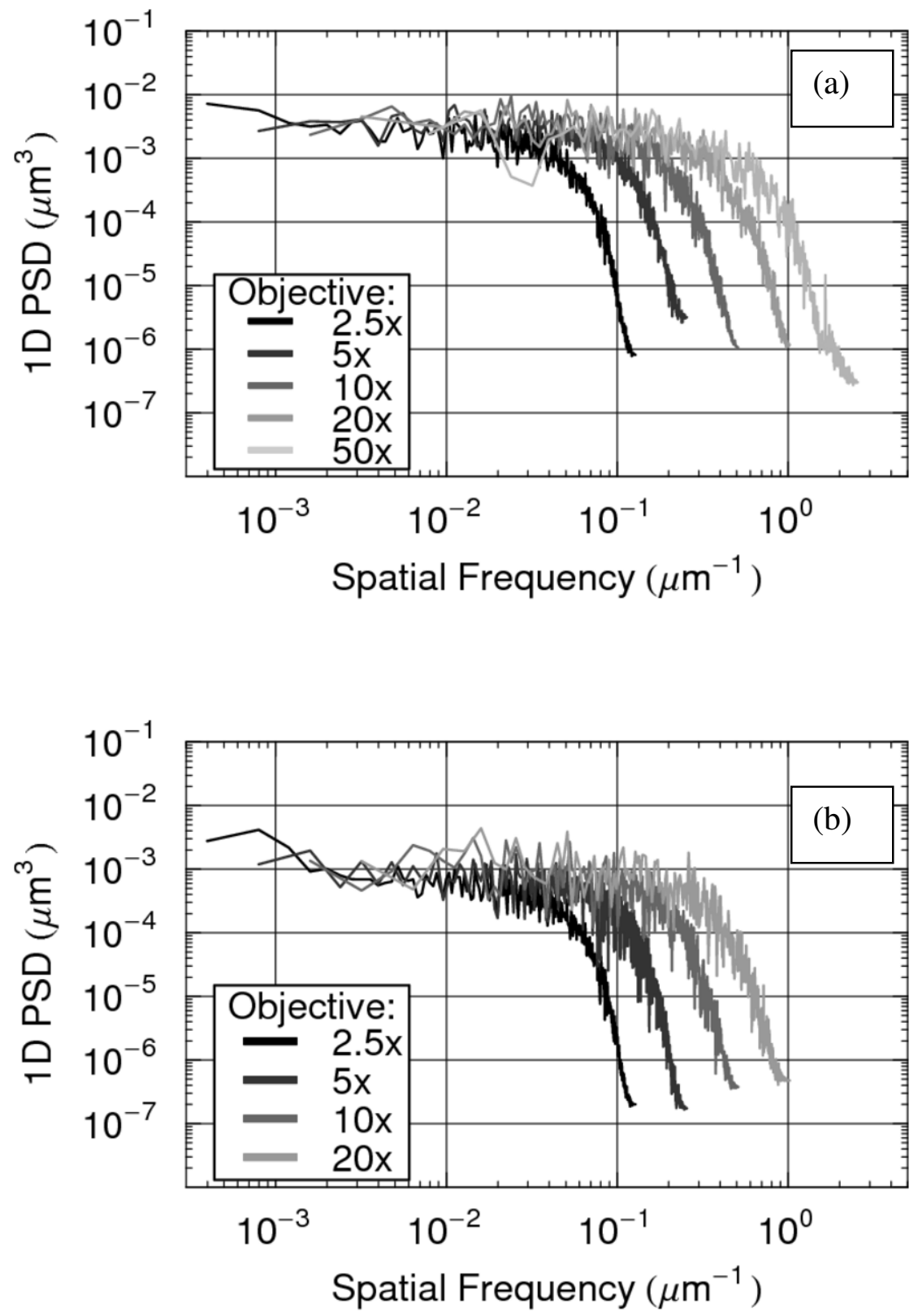

Figure 6. The comparison of the undercut sidewall $\mathrm{SF}_{6}$ etched sample (a) with the essentially vertical sidewall $\mathrm{HBr}$ sample (b). Even with the $\mathrm{SF}_{6}$ etched BPRA, the PSD of the surface deviates from flat by at most $\% 10$. Note that the $50 \times$ objective data is not available for the $\mathrm{HBr}$ etched sample. For the objectives measured, the $\mathrm{HBr}$ etched BPRA has essentially an ideal profile and flat PSD beyond the frequencies of interest. 


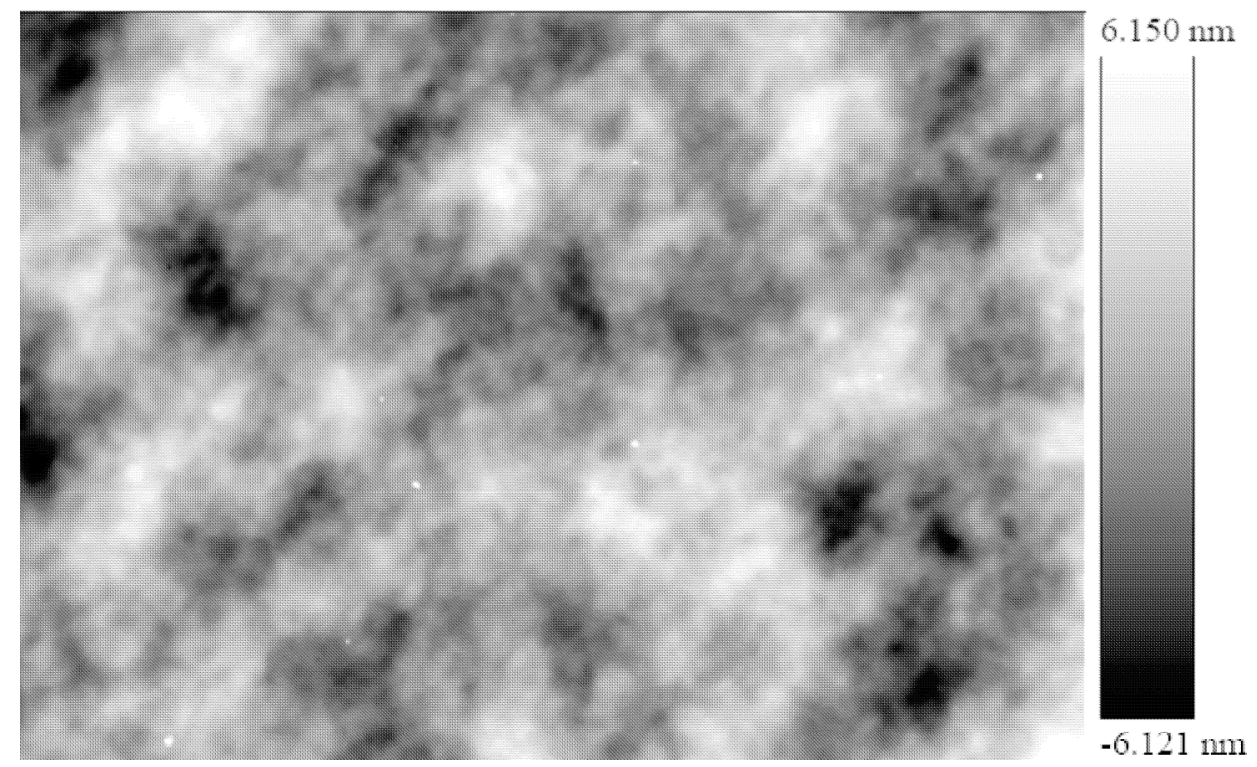

(a)

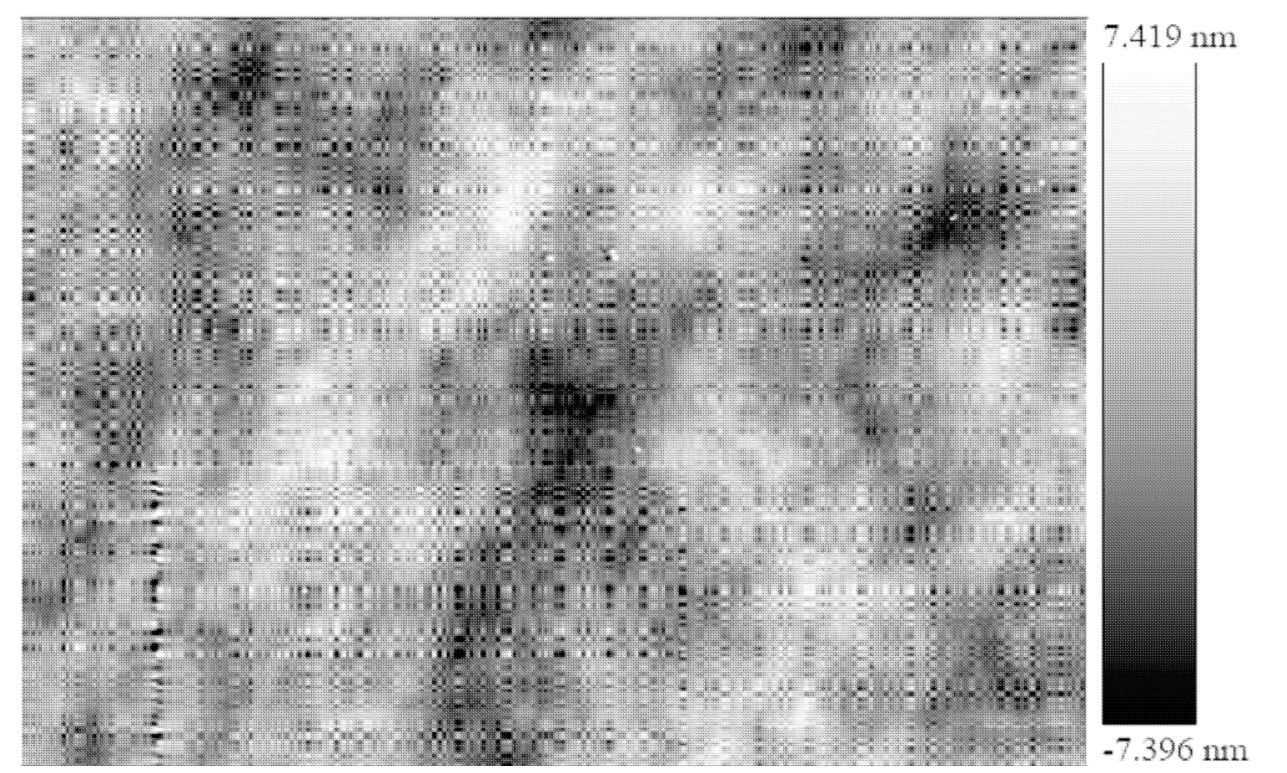

(b)

Figure 7. Micromap ${ }^{\mathrm{TM}}-570$ measurements with the $2.5 \times$ objective of the bare silicon surface (a) and shallow $49 \mathrm{~nm}$ etch depth $600 \mathrm{~nm}$ BPRA (b). The large-scale roughness of a standard silicon "prime" wafer is evident at lower spatial frequencies. The low frequency roughness limits the use of these reference samples in calibrating the $2.5 \times$ and $5 \times$ objectives for high accuracy. Polished silicon flats should not have this roughness. 
